\title{
Myomaker and Myomixer Characterization in Gilthead Sea Bream Under Different Myogenesis Conditions.
}

\author{
Miquel Perelló-Amorós \\ University of Barcelona \\ Aitor Otero-Tarrazón \\ University of Barcelona \\ Violeta Jorge-Pedraza \\ University of Barcelona \\ Isabel García-Pérez \\ University of Barcelona \\ Albert Sánchez-Moya \\ University of Barcelona \\ Jean-Charles Gabillard \\ National Research Institute for Agriculture, Food and Environment \\ Fatemeh Moshayedi \\ University of Barcelona \\ Isabel Navarro \\ University of Barcelona \\ Encarnación Capilla \\ University of Barcelona \\ Jaume Fernández-Borràs \\ University of Barcelona \\ Josefina Blasco \\ University of Barcelona \\ Chillarón Josep \\ University of Barcelona \\ Daniel García de la serrana \\ University of Barcelona \\ Joaquim Gutiérrez ( $\nabla$ jgutierrez@ub.edu ) \\ University of Barcelona
}

Research Article

Keywords: Myomaker, Myomixer, myogenesis, muscle regeneration, muscle growth, fish. 
Posted Date: December 28th, 2021

DOI: https://doi.org/10.21203/rs.3.rs-1123861/v1

License: (c) (i) This work is licensed under a Creative Commons Attribution 4.0 International License. Read Full License 


\section{Abstract}

The skeletal muscle is formed by multinucleated myofibers originated by waves of hyperplasia and hypertrophy during myogenesis. Tissue damage triggers a regeneration process including new myogenesis and muscular remodeling. During myogenesis, the fusion of myoblasts is a key step that requires different genes' expression, including the fusogens Myomaker and Myomixer. The present work aimed to characterize these proteins in gilthead sea bream and their possible role in in vitro myogenesis, at different fish ages and during muscle regeneration after induced tissue injury. Myomaker is a transmembrane protein highly conserved among vertebrates; whereas Myomixer is a micropeptide that is moderately conserved but maintains its crucial AxLyCxL motif. myomaker expression is restricted to skeletal muscle, while the expression of myomixer is more ubiquitous. In primary myocytes culture, myomaker and myomixer expression peaked at day 6 and day 8, respectively. During regeneration, the expression of both fusogens and all the myogenic regulatory factors showed a peak after 16 days post-injury. Moreover, myomaker and myomixer were present at different ages, but in fingerlings there were significantly higher transcript levels than in juveniles or adult fish. Overall, Myomaker and Myomixer are valuable markers of muscle growth that together with other regulatory molecules, can provide a deeper understanding of myogenesis regulation in fish.

\section{Introduction}

The skeletal muscle is a large and complex tissue formed by long multinucleated cells called myofibers that are the functional units of the locomotor system in vertebrates. The process by which the skeletal muscle is formed is known as myogenesis, where mesenchymal stem cells are committed to the muscle lineage as myoblasts, undergoing a process that involves proliferation and cell fusion events ${ }^{1}$. Such complex cellular process is finely regulated by a series of highly conserved master transcription factors, known as myogenic regulatory factors (MRFs), which coordinate the expression of all the required molecular machinery and structural components of the muscle. This myogenic program can occur during the embryonic development of the muscle, but also along the adulthood in response to challenging conditions or tissue damage ${ }^{2}$ ). Hence, in animal production, to have a complete understanding of myogenesis may help to improve muscle growth, its remodeling and recovery after an injury. This knowledge is a base ground for further research towards a better flesh quality and thus, an improvement in the production of healthy protein sources for human consumption. In fish, in contrast to mammals, myogenesis extends beyond the adult stage due to an indeterminate and continuous growth throughout their lives, which is made possible by mechanisms of hyperplasia and hypertrophy ${ }^{3}$.

The MRFs, as well as other genes from the paired box (Pax) and SRY-related HMG-box (Sox) family, control the expression of structural proteins, such as myosin heavy chain (Mhc) or the proteins that permit myoblast fusion, like the recently discovered muscle-specific proteins Myomaker and Myomixer. In vertebrates, muscle differentiation is based on the sequential activation of the MRFs, Pax and Sox molecules: first, the Myogenic Factor 5 (Myf5), the Myogenic Differentiation 1 Protein (MyoD) and Sox8 specify the myoblast for differentiation; then, Myogenin, the Myogenic Factor 6 (Mrf4) and Pax7 participate 
in the late differentiation process and trigger the expression of myotube specific genes to form the multinucleated myofibers ${ }^{4}$.

The muscle specific Myomaker and Myomixer proteins that participate in the regulation of myogenesis play a central role in cellular fusion, development, and regeneration of mammalian muscle ${ }^{5}$. Myomaker, first known as Tmem8c, is a highly conserved transmembrane protein in vertebrates. In mammals, myomaker encodes for a 221 amino acids (aa) protein ${ }^{6}$, while in fish it has more variation. For instance, in rainbow trout (Oncorhynchus mykiss) Myomaker encodes for 434 aa and in zebrafish (Danio rerio) for 221 aa 7,8 . Although most of the reported experiments were done in mammals, in all vertebrates studied, including fish, myomaker expression is fundamental in both embryogenesis and regeneration of adult skeletal muscle ${ }^{7-13}$. The expression pattern of myomaker is similar to myod and myogenin because its regulation is in fact carried out through these MRFs ${ }^{14}$.

Indeed, the transcription factors MyoD and Myogenin bind to the two E-boxes present in the promoter of the myomaker gene to induce its expression ${ }^{11,14}$. Several studies in mice have shown that myomaker expression was maximal during myoblast fusion and that the loss of myomaker inhibited cell fusion during myogenesis ${ }^{10}$. Thus, all these data confirm the involvement of Myomaker in this process of myotubes formation. In fish, most of this research was done in salmonids or zebrafish. In rainbow trout, myomaker is expressed during embryogenesis and muscle regeneration after an injury, where the maximal expression is at the stage of the myoblast fusion ${ }^{8}$, and in zebrafish, myomaker expression is restricted to embryogenesis 7. Thus, further studies in non-salmonid fish are needed to understand the role of Myomaker in myogenesis and in adult teleost muscle regeneration.

Myomixer, also called Myomerger or Minion, is a weakly conserved transmembrane protein in vertebrates. In mammals, myomixer encodes for an 84 aa protein ${ }^{15}$. In fish, Myomixer is a 75 aa protein in zebrafish ${ }^{15}$ and a 77 aa peptide in rainbow trout ${ }^{13}$. myomixer is expressed during embryogenesis, muscle regeneration and myoblast differentiation in mice and trout 9,13,15. Similarly to myomaker, MyoD and Myogenin regulate the transcription of myomixer by binding to the three E-boxes of its promoter during myogenesis ${ }^{12}$. A recent study observed that the lack of myomixer produced a defect in the process of cell fusion throughout myogenesis, confirming the involvement of Myomixer during myoblast fusion ${ }^{9}$.

The expression pattern during embryogenesis and muscle regeneration of both, myomaker and myomixer is very similar, which originated an initial theory of a physical interaction between both proteins (Reviewed by Chen and coworkers ${ }^{5}$ ). Nevertheless, recent bibliography demonstrated that knocking out either myomaker or myomixer completely impaired myoblast fusion, but not myoblast differentiation, thus the coexistence of Myomaker (in both cells that are going to fuse) and Myomixer (in at least one of the cells) is necessary and enough to promote cell fusion. However, Chen and coworkers ${ }^{5}$ later refuted the theory of the direct physical interaction between Myomaker and Myomixer, then having both proteins essential, but independent roles in myoblast fusion in the studied species ${ }^{5}$. 
Muscle regeneration, in vitro myogenesis or the comparison of different growth stages, offer useful models to approximate to the functions of MRFs, Myomaker and Myomixer and their relationships in muscle growth regulation. Thus, the objective of this study was first to characterize Myomaker and Myomixer genes in gilthead sea bream, and second, to investigate their expression during in vivo muscle regeneration after induced injury, throughout in vitro myogenesis and, at different stages of fish muscle growth.

\section{Materials And Methods}

\subsection{Fish maintenance and distribution}

In order to perform the muscle regeneration experiment, 140 gilthead sea bream (Sparus aurata) juveniles (initial body weight: $15.4 \pm 3.5 \mathrm{~g}$; initial length: $8.7 \pm 0.6 \mathrm{~cm}$ ) were obtained from a commercial hatchery (Piscimar, Borriana, Spain) and were placed and adapted to the fish facilities of the Faculty of Biology (University of Barcelona). Fish were randomly distributed in three $200 \mathrm{~L}$ seawater tanks ( $46-47 \mathrm{fish} / \operatorname{tank})$. Four gilthead sea bream adults of $214.0 \pm 12.13 \mathrm{~g}$ were reared in one $200 \mathrm{~L}$ tank for a tissue screening analysis of Myomaker and Myomixer and additionally, three groups of eight gilthead sea breams weighing $5.88 \pm 0.51 \mathrm{~g} ; 122.38 \pm 2.31 \mathrm{~g}$ and $387.13 \pm 41.9 \mathrm{~g}$ more were reared in three $200 \mathrm{~L}$ tanks for an ontogenetic study of the expression of both genes. Each tank had a constant flux of $700 \mathrm{~L} / \mathrm{h}$ in a seawater semi-closed recirculation system with a weekly water renewal of $20-30 \%$, a salinity of $35-37 \%$, a constant temperature of $23 \pm 1^{\circ} \mathrm{C}$ and a photoperiod of $12 \mathrm{~h}$ light/12 $\mathrm{h}$ dark. Fish were fed ad libitum 3 times per day (9 a.m., 2 p.m. and 7 p.m.) with a commercial diet (Perla, Skretting, Burgos, Spain) and were kept in the described conditions for the acclimation period during 2 weeks before the experiments. The study was carried out following the EU recommendations and the procedures established by the Spanish and Catalan governments. All the experimental designs included within this manuscript fulfills the ARRIVE guidelines (https://arriveguidelines.org/arrive-guidelines). The protocol was approved by the Ethics and Animal Care Committee of the University of Barcelona (CEEA OB72/17).

\subsection{Myomaker and Myomixer characterization}

The myomakermRNA (CDNA) sequence of $S$. aurata (XM_030418477.1) was obtained from the GenBank (https://www.ncbi.nlm.nih.gov/genbank/) and the myomixer sequence was found in the CCMAR Sequence Server database ${ }^{16}$. The myomaker and myomixer cDNA sequences were blasted against the gilthead sea bream genome deposited in Ensembl (https://www.ensembl.org/Sparus_aurata/Info/Index) to obtain the genomic sequence of both genes for its characterization. Primers for the amplification by real-time quantitative PCR (qPCR) of myomaker and myomixer cDNA (Table 1) were designed using the sequences mentioned above with the Primer3Plus software (http://www.bioinformatics.nl/cgi-

bin/primer3plus/primer3plus.cgi). The forward primer of myomaker was designed in the exon 1-exon 2 junction to avoid amplification of genomic DNA and the reverse primer was placed in exon 2. The primers of myomixer were designed on its single exon, so DNase I pretreatment of the RNA was necessary before reverse transcription. The quality of the primers was tested by using the NetPrimer software (http://www.premierbiosoft.com/netprimer/). The collection of myomaker and myomixer sequences from different species was performed through the BLAST databases (https://blast.ncbi.nlm.nih.gov/Blast.cgi). 
The Unipro UGENE v33.0 software was used to obtain the predicted protein sequences from the nucleotide sequences. Multiple Myomaker and Myomixer sequence alignments were performed with the MAFFT tool (https://mafft.cbrc.jp/alignment/server/). The iterative refinement method L-INS-i was used for the Myomaker sequence and the progressive method G-INS-1, for the Myomixer sequence. In both cases, a gap opening penalty of 1.53 (default settings) was used. The alignments were confirmed with the Unipro UGENE v33.0 software. The phylogeny was developed with the Maximum Likelihood phylogenetic inference method of the MEGA X v10.1.7 program. The JTT+G protein substitution model was used with a bootstrap value of 500 .

\subsection{Tissue screening}

Four $214.0 \pm 12.13 \mathrm{~g}$ gilthead sea breams were deprived of food over-night, anesthetized with MS222 (100 $\mathrm{mg} / \mathrm{l}$ ) and weighed. Then, blood was drawn from the caudal vein with a $1 \mathrm{ml}$ sterile syringe and a 0.4- or 0.6-mm needle previously precoated with EDTA-Li to prevent blood clotting. For tissue collection, fish were slaughtered by cervical section of the spine and the following tissues were extracted: white muscle, red muscle, skin, heart, brain, adipose tissue, liver, spleen, hypophysis, kidney, gill, intestine, bone, pyloric caeca, stomach, and gonad. The tissues were introduced in RNase-free microtubes that were stored during sampling in liquid nitrogen and at the end at $-80^{\circ} \mathrm{C}$ until further analysis.

\subsection{In vitro myogenesis: primary myoblast culture}

To study in more detail the role of Myomaker and Myomixer along with the MRFs during the myogenic process, the expression of both genes was analyzed during the course of the primary myoblast culture from the proliferative stage (days 0 to 4 ) and through the complete differentiation process (day 4 to 12 ). The primary satellite cells from gilthead sea bream skeletal white muscle were isolated and cultured as myoblasts as previously described ${ }^{17-19}$. A total of six independent cell isolations were performed as biological replicates and samples for gene expression were taken every two days after the satellite cells seeding.

\subsection{Muscle regeneration experiment}

The muscle regeneration experiment aimed to better understand the role of Myomaker and Myomixer in myogenesis after muscle injury. To do that, 140 gilthead sea bream were divided into two groups: injured fish (I) and control fish (C). First, gilthead sea bream juveniles were all anesthetized with MS222 (100 mg/l) and then measured and weighed. To identify the fish, a passive integrated transponder (PIT) tag (ID100A(1.25) Nano transponder; Trovan Electronic Identification Systems, Madrid, Spain) was inserted subcutaneously into the left anterior epaxial muscle just below the first radius.

Subsequently, an injury was performed with a $2.108 \mathrm{~mm}(14 \mathrm{G})$ diameter needle inserted vertically into the left epaxial muscle below the sixth radius to a depth of $1 \mathrm{~cm}$. To know exactly where the needle was introduced, the tip of the sixth radius was cut. Then, the wound was healed with iodine alcoholic solution and the fish was allowed to recover in a separated small tank before to be returned to its original tank. 
Samplings were done at days $0,1,2,4,8,16$ and 30 after the injury, in which white muscle was extracted. At each time point, fish were deprived of food over-night and 20 fish were randomly selected for sampling (4-5 injured fish/tank and 2 control fish/tank). Fish were first anesthetized, identified reading the pig tag, weighed to note the changes on body weight and then, blood was drawn. For tissue extraction, all fish were slaughtered as mentioned before. In injured fish, a section of the muscle was removed from the left side (injured), while the right side was also taken as a self-control for each fish. The size of the muscle extracted was $0.5 \mathrm{~cm}$ wide and $1 \mathrm{~cm}$ long just below the cut radius. All tissue samples were placed in RNase-free microtubes, which were stored in liquid $\mathrm{N}_{2}$ during sampling and then at $-80^{\circ} \mathrm{C}$ until further analysis.

\subsection{RNA extraction and cDNA synthesis}

For RNA extraction, $1 \mathrm{~mL}$ of TRI Reagent Solution $®$ (Applied Biosystems, Alcobendas, Spain) was added to the samples (around $0.04 \mathrm{~g}$ for liver and $0.1 \mathrm{~g}$ for the rest of the tissues, whenever possible). Samples were homogenized with the Precellys Evolution ${ }^{\circledR}$ (Bertin Instruments, Montigny-le-Brettoneux, France) adjusting the protocol depending on the hardness and elasticity of the tissue. Here below, RNA extraction was performed following the manufacturer's instructions of the TRI Reagent Solution ${ }^{\circledR}$. The final concentration of each sample was obtained using a Nanodrop $2000^{\mathrm{TM}}$ (Thermo Scientific, Alcobendas, Spain). RNA integrity was confirmed in a $1 \%$ agarose gel $(\mathrm{m} / \mathrm{v})$ stained with SYBR-Safe DNA Gel Stain ${ }^{\circledR}$ (Life Technologies, Alcobendas, Spain). For cDNA synthesis, $1 \mu \mathrm{g}$ of total RNA was treated with DNase I Amplification Grade ${ }^{\circledR}$ (Life Technologies, Alcobendas, Spain) to remove all genomic DNA. Reverse transcription was carried out with the First Strand cDNA synthesis Transcriptor Kit ${ }^{\circledR}$ (Roche, Sant Cugat del Valles, Spain) following the manufacturer's recommendations. According to the requirements of the MIQE guidelines ${ }^{20}$, the mRNA transcripts levels of the genes were analyzed by qPCR using the CFX $384^{\mathrm{TM}}$ RealTime System (Bio-Rad, El Prat de Llobregat, Spain). The analysis was performed in a final volume of $5 \mu \mathrm{L}$, containing $2.5 \mu \mathrm{L}$ of iTaq SYBR Green Supermix ${ }^{\circledR}$ (Bio-Rad, El Prat de Llobregat, Spain), $0.125 \mu \mathrm{L}$ of forward $(250 \mathrm{nM})$ and reverse $(250 \mathrm{nM})$ primers, $1 \mu \mathrm{L}$ of cDNA from each sample and $1.25 \mu \mathrm{L}$ of DEPC water. The reaction was performed in triplicate in 384-well plates (Bio-Rad, El Prat de Llobregat, Spain) under the conditions described by Salmerón and coworkers ${ }^{21}$. The qPCR consisted of 1 ) an activation phase of $3 \mathrm{~min}$ at $\left.95^{\circ} \mathrm{C} ; 2\right) 40$ cycles of $10 \mathrm{~s}$ at $95^{\circ} \mathrm{C}$ and $30 \mathrm{~s}$ at $55-68^{\circ} \mathrm{C}$ (dependent of the melting temperature of the primers, Table 1); and 3) a melting curve from $55^{\circ} \mathrm{C}$ to $95^{\circ} \mathrm{C}$ that increased by $0.5^{\circ} \mathrm{C}$ every $30 \mathrm{~s}$. Before this analysis, the adequate cDNA dilution for each gene was determined by a dilution curve with a pool of samples. With this analysis, the specificity of the amplification, the absence of primersdimers and the efficiency of the primers were also tested. 
Table 1

Primers used in the Real-Time quantitative PCR analyses.

\begin{tabular}{|c|c|c|c|}
\hline Gene & Primer sequences $\left(5^{\prime}-3^{\prime}\right)$ & $\mathrm{Ta}\left({ }^{\circ} \mathrm{C}\right)$ & Accession Number \\
\hline \multirow[t]{2}{*}{ myf5 } & F: CCATCCAGTACATCGAGAGCC & 57 & KJ524459 \\
\hline & R: ATCGCCCAAAGTGTCGTTCT & & \\
\hline \multirow[t]{2}{*}{ myod1 } & F: TTTGAGGACCTGGACCC & 60 & AF478568.1 \\
\hline & R: CTTCTGCGTGGTGATGGA & & \\
\hline \multirow[t]{2}{*}{ myod2 } & F: CACTACAGCGGGGATTCAGAC & 60 & AF478569 \\
\hline & R: CGTTTGCTTCTCCTGGACTC & & \\
\hline \multirow[t]{2}{*}{$m r f 4$} & F: CATCCCACAGCTTTAAAGGCA & 60 & JN034421 \\
\hline & R: GAGGACGCCGAAGATTCACT & & \\
\hline \multirow[t]{2}{*}{ myogenin } & F: CAGAGGCTGCCCAAGGTCGAG & 68 & EF462191 \\
\hline & R: CAGGTGCTGCCCGAACTGGGCTCG & & \\
\hline \multirow[t]{2}{*}{ myomaker } & F: TTCACTGCGGTTTACCACGC & 60 & XM_030418477.1 \\
\hline & R: CCCACATAGAGAGAGCTGTGCTG & & \\
\hline \multirow[t]{2}{*}{ myomixer } & F: TGCTGCGGTCCCTGGTTATC & 60 & LR537135.1 \\
\hline & R: ACTCCTGGGATCGAATGCGG & & \\
\hline \multirow[t]{2}{*}{ ef1a } & F: CTTCAACGCTCAGGTCATCAT & 60 & AF184170 \\
\hline & R: GCACAGCGAAACGACCAAGGGGA & & \\
\hline \multirow[t]{2}{*}{ rps18 } & F: TGACGGAAGGGCACCACCAG & 60 & AY550956 \\
\hline & R: AATCGCTCCACCAACTAAGAACGG & & \\
\hline \multirow[t]{2}{*}{ rp/27a } & F: AAGAGGAACACAACTCACTGCCCCAC & 60 & AY188520 \\
\hline & R: GCTTGCCTTTGCCCAGAACTTTGTAG & & \\
\hline
\end{tabular}

The expression level of each gene was calculated with the Pfaffl method ${ }^{22}$ and was analyzed relative to the geometric mean of the reference genes ( $r p s 18, r p / 27$ and ef1a). The reference genes, the most stable under different conditions, were confirmed with the GrayNorm algorithm.

\subsection{Statistical analyses}

Data were analyzed using IBM SPSS Statistics v. 25 and were presented as mean \pm standard error of the mean (SEM). Normal distribution was analyzed using the Shapiro-Wilk test and homogeneity of the 
variances (homoscedasticity) was assessed with Levene's test. If normal distribution and/or homoscedasticity was not found, data were transformed logarithmically. Differences were tested by Student's t-test or one-way analysis of variance (ANOVA) and the post-hoc Tuckey HSD. If necessary, the nonparametric Kruskal Wallis test and the post-hoc T3 de Dunnett were used. Additionally, one-way ANOVA was performed to verify that the tank did not influence the measured parameters. Statistical differences were considered significant when $p<0.05$.

\section{Results}

\subsection{Myomaker and Myomixer characterization}

A search in GenBank was performed to identify the gilthead sea bream myomaker mRNA (XM_030418477.1) while the genomic sequence of myomaker was found in the gilthead sea bream genome deposited in the Ensembl (ENSSAUG00010019449). Two ancient paralogues of myomakerwere identified, named pgap6 (ENSSAUG00010014619) and tmem8b (ENSSAUG00010020348), with low homology to myomaker, 33.19 and $30.60 \%$, respectively. This search also revealed a single transcript (ENSSAUT00010049102.1) that apparently contained only 5 exons encoding a 232 aa protein. However, the alignment between the cDNA and the genomic sequence revealed that the automatic exon finding algorithm of the Ensembl included the exon 6 sequence inside the $3^{\prime}$ UTR. Thus, it was determined that the myomaker gene was situated in chromosome 5 and contained 6 exons encoding a protein of 285 aa (Figure 1). The gilthead sea bream Myomaker protein (XP_030418477.1) shared 89.50\% identity with the zebrafish Myomaker (NP_001002088.1) protein; 81.75\% identity with the rainbow trout Myomaker protein (XP_021476828.1) and 71.56\% with the mouse Myomaker protein (NP_079652.1).

The gilthead sea bream myomixer sequence was provided by the CCMAR Sequence Server. The cDNA sequence of myomixer was blasted against the gilthead sea bream genome deposited in the Ensembl to find its genomic sequence (ENSSAUG00010011859, no paralogs identified). The myomixer gene is located in the chromosome 15 and has one single transcript (ENSSAUT00010028952.1) containing only 1 exon encoding a 75 aa protein. The gilthead sea bream Myomixer protein shares $70.67 \%$ identity with the zebrafish Myomixer protein (PODP88.1); 69.33\% with the rainbow trout Myomixer protein (QII57370.1) and $33.33 \%$ with the mouse Myomixer protein (NP_001170939.1).

The phylogenetic analysis of the Myomaker and Myomixer aa sequences is shown in the figures 2 and 3 . In both cases, a clear evolution of the proteins is observed across vertebrates, from fish to mammals. The Myomaker and Myomixer gilthead sea bream sequences are more closely related to other perciformes, such as other Sparidae species (Acanthopagrus latus) and other species such as the European sea bass (Dicentrarchus labrax). Myomixer diverged notably more than Myomaker. Moreover, the Myomaker protein sequences in fish have a great disparity in length (Figure 2). In gilthead sea bream, Myomaker is a 285 aa protein, while in salmoniforms it ranges from 400 aa in brown trout (Salmo trutta) and up to 477 in chinook salmon (Oncorhynchus tshawytscha). In chondrichthyans, the sequence of Myomaker is the smallest, having 218 aa similar to the Myomaker protein sequences in terrestrial vertebrates (amphibians, reptiles, birds and mammals), which vary between 220 and 221 aa. 
In contrast, the length of the Myomixer protein sequences (Figure 3) diverged less than Myomarker among the different vertebrate species. In most fish, Myomixer has 75 aa, as in S. aurata. However, some salmonids, such as 0 . kisutch, have a sequence of 99 aa. In terrestrial vertebrates, the sequences range from 62 aa in birds to 108 aa in the reptile Paroedura picta. In mammals, Myomixer remains at 84 aa. Nevertheless, the multiple sequence alignment highlighted that the crucial AxLyCxL motif of the Myomixer is highly conserved across the vertebrates (data not shown).

\subsection{Myomaker and Myomixer tissue screening}

The Figure 4 shows the myomaker and myomixer gene expression in gilthead sea bream tissues. The myomakergene was mostly expressed in white and red muscle at similar levels while in the rest of the tissue's mRNA levels were very low. In contrast, the myomixer gene showed expression in white and red muscle, as well as, in skin, heart, brain, adipose tissue, bone, and gonad with relatively high levels. Both the myomaker and myomixer transcript levels in these tissues were also confirmed in the agarose gel after a qPCR. In the other tissues, the expression of myomixer was insignificant.

\subsection{Regeneration Study \\ 3.3.1. Myomaker and Myomixer}

The Figure 5 shows a comparative expression profile of myomaker $(\mathrm{A})$ and myomixer $(\mathrm{B})$ genes during the regeneration period in white muscle of gilthead sea bream from time of injury (day 0 ) to 30 days later. Thus, after a stable period with almost constant values until day 8 a significant peak (3-fold) at day 16 was observed following a decrease up to day 30 .

\subsubsection{MRFs}

The different myogenic genes (myod1, myod2, myf5, myogenin and mrf4,) presented a similar profile during muscle regeneration with a maximum peak of expression 16 days after injury, maintaining high levels up to day 30 (Figure 6A-E).

\subsection{In vitro myogenesis}

\subsubsection{Myomaker and Myomixer}

The expression of myomaker and myomixer increased significantly already at day 4 of culture during in vitro myogenesis of gilthead sea bream, reaching at day 6 its maximum level, followed by a progressive decrease, significant at day 12 (Figure 7).

\subsubsection{MRFs}

myod 1 and myod 2 gene expression levels presented their earliest peak, at day 4 and day 6 respectively. Then, in the case of myod 1 , levels progressively diminished, being the decrease significant at day 6 and reaching the lowest values of expression at day 12. myod2 expression decreased after its peak although not significantly, maintaining a plateau until day 12 (Figure 8A-B). Otherwise, myf5 increased steadily starting day 2 reaching significant differences at day 10 (Figure 8C). 
myogenin expression increased rapidly showing significant changes at day 4 , followed by a progressive decrease from day 6 until day 12 (Figure 8D). The mrf4 expression showed a tendency to increase already at day 4 , although it was only significant at day 10 (Figure 8E).

\subsection{Myomaker and Myomixer at different growing stages}

The Figure 9 shows the expression of myomaker (A) and myomixer (B) in white muscle of fingerlings, juveniles, and adults of gilthead sea bream. Both genes showed a progressive decrease with the age of the fish. Thus, fingerlings presented the maximum gene expression levels, in juveniles those decreased significantly, and adults showed very low levels of myomaker and myomixer although differences in gene expression were only significant in comparison to fingerlings.

\section{Discussion}

The muscle specific Myomaker protein that controls myoblast fusion was initially found in mice as a 221 aa protein, and it was described to have a similar transcription profile as those of myod and myogenin ${ }^{10}$. In fish, Myomaker was first described in zebrafish ${ }^{7}$, and recently it has been characterized in rainbow trout 8 and yellowfin seabream (Acanthopagrus latus) ${ }^{23}$. In all the three species, the gene is structured in 6 exons, but differences in the length of the protein are marked, being as long as 434 aa in rainbow trout 8 , while just 285 aa in non-salmonid species such as yellowfin seabream ${ }^{23}$ and gilthead seabream. The protein sequence alignment between mice, rainbow trout and gilthead seabream, pointed out that the Nterminal half of the rainbow trout Myomaker was similar to the mice and gilthead seabream sequences, while the $\mathrm{C}$-terminal half did not have homology with any known motifs ${ }^{8}$. The phylogenetic analysis showed that Myomaker is a well conserved protein across vertebrate organisms, from fish to mammals 8,10 . The gilthead sea bream Myomaker protein presented a homology of $89 \%, 81 \%$ and $71 \%$ with zebrafish, rainbow trout and mouse, respectively. Moreover, a clear evolution among fish species was observed. and the gilthead sea bream Myomaker sequence resulted more closely related to other perciform species, such as the European sea bass ( $D$. labrax), the beloniform species, such as 0 . latipes, or the salmonids.

The fusogenic gene myomixer was also firstly identified in mice, contained a single exon and encoded an 84 aa protein. Additionally, the mice myomixer gene had another transcript form, less conserved, which had 3 exons and yielded a protein of 108 aa ${ }^{9,12}$, but until today, a single myomixertranscript has been described in the few fish species where this gene has been studied. Indeed, the only described gilthead sea bream myomixer transcript (ENSSAUT00010028952.1) that was found in the gilthead sea bream genome deposited in Ensembl contained only one exon that encoded a 75 aa protein, similarly to the rainbow trout myomixer (77 aa), although in this salmonid species, the gene is structured in two exons ${ }^{13}$.

The Myomixer protein sequence showed weak cross-species conservation, with mammals and fish sharing only $36 \%$ identity ${ }^{13,15}$. Among fish, the Myomixer gilthead sea bream showed an homology of $70.67 \%$ and $69.33 \%$ with the zebrafish and rainbow trout Myomixer protein sequences, respectively (unpublished data). Nevertheless, the crucial AxLyCxL motif of the Myomixer micropeptide presented a high conservation across vertebrates. As in the case of Myomaker, the gilthead sea bream Myomixer sequence was more 
related to that from other perciformes, such as the European sea bass, beloniformes or salmoniformes. Other phylogenetic analysis performed in gilthead sea bream, such as those for Myogenin and Preproghrelin proteins also showed that these molecules evolved in the same way, being closer to other perciforms, while being more distant to salmoniformes or cypriniformes ${ }^{24}$.

Regarding the tissue screening, on one side, the gene expression of myomaker in gilthead sea bream showed a narrow distribution among tissues, being expressed mainly in white and red skeletal muscles. Such expression distribution was also observed in rainbow trout ${ }^{8}$ and yellowfin seabream ${ }^{23}$. On the other hand, the myomixer pattern of expression in adult gilthead sea bream was not restricted to white and red muscles, thus differing from the findings in rainbow trout and mice ${ }^{12,13}$.

While in mammals, regenerative myogenesis is a well-known process, in fish, some aspects remain unclear. Regeneration in gilthead sea bream after muscle injury was first studied by Rowlerson and coworkers ${ }^{25}$. The histological analysis showed a high cellular proliferation with a greater deposition of connective tissue and new small myofibers formation around the lesion site by 7-11 days after the injury was made. In rainbow trout muscle, after 20 and 30 days of a mechanical injury, an alteration of the muscle fiber organization was found at the site of damage due to a high deposit of connective tissue with small muscle fibers ${ }^{8,26}$. The regenerative process is not complete at that time and coincides with the onset of a peak of myogenin, myomaker and myomixer expression ${ }^{8,27}$. The differences observed between the two species regarding the moment when the new myofibers were formed during myogenesis could be due to the distinct metabolic rates, being higher in the gilthead sea bream, reared at $21-23^{\circ} \mathrm{C}$, compared to the rainbow trout, reared at $10-15^{\circ} \mathrm{C} 8,13,28$. At day 1 , the injury was easily observed with the naked eye, while on days 8 and 16, the muscle damage was no longer visually noticeable (pictures shown in Supplementary Material). This could mean that new myofibers would already be forming to repair the muscle injury. At a transcriptional level, there were appreciable differences between rainbow trout and gilthead sea bream indicating also a faster regenerative process in the latter. In the current study, the expression of MRFs, along with the myomaker and myomixerfusogens was evaluated through the regenerative process. In mice, both myomaker and myomixer expression was strongly detected in regenerating muscle 3 days after the injury and then rapidly decreased in less than 2 days when the new myofibers were formed, which indicated that both proteins are essential for muscle regeneration 9,10 . The regulation of the expression of both genes is mediated by two E-boxes in the promoter, which are described as targets of MyoD and Myogenin ${ }^{14}$. Furthermore, specifically knocking out myomaker in the mice satellite cells in vivo completely impaired myoblast fusion, thus resulting in a complete blocking of muscle regeneration ${ }^{11}$.

In fish, the implication of Myomaker and Myomixer in muscle regeneration has only been studied recently in rainbow trout, where the expression of both genes, drastically increased only at 30 days post injury, along with myogenin ${ }^{8,13}$. Such response coincides with the observations in injured mice models where, although the regeneration process occurs in a much shorter time, the expression of both myomaker and myomixer peaked at 3 days post injury ${ }^{5}$. In the present study, the muscle regeneration experiment in gilthead sea bream showed that myomaker, myomixer and all the MRFs were strongly upregulated at day 16 , while most of the genes were decreasing at day 30 , thus presenting the gilthead sea bream a faster 
response than that observed in trout, where the analyzed genes were upregulated only after 30 days of recovery ${ }^{8}$. Moreover, another aspect that could affect the metabolic rate, in addition to the temperature at which the fish are reared, is the influence of fish size. In the regeneration experiment with rainbow trout performed by Landemaine and coworkers ${ }^{8}$ the authors used $1 \mathrm{~kg}$ fish, in comparison with the $15 \mathrm{~g}$ gilthead sea bream that were utilized in the present study. In general, smaller fish shows higher metabolic rates and therefore, faster muscle regeneration. Moreover, the present findings are in agreement with those of Rowlerson and coworkers ${ }^{25}$ by histological studies in gilthead sea bream, as the new small myofibers were deposited in the lesion site around day 8 post-injury, at the same moment that it was observed a significant increase in MRFs, myomaker and myomixer gene expression. Overall, these data support the importance of both, Myomaker and Myomixer in the regenerative process of skeletal muscle in gilthead sea bream.

The in vitro study performed showed that myomaker presented a peak of expression levels at day 6 decreasing later progressively, while for myomixer, the highest expression was found at day 8 This temporal distribution is in agreement with the previously described role of both molecules ${ }^{5}$. Thus, the action of Myomaker inducing the hemifusion of the membranes would happen slightly earlier than that of Myomixer, which is the formation and expansion of the pore between the plasma membranes ${ }^{5}$. The current results coincide with those obtained in rainbow trout in vitro myoblasts, where the expression of both myomaker and myomixer was increasing progressively during differentiation stages 8,13 .

The MRF expression during in vitro gilthead sea bream myogenesis was well described by our group ${ }^{17}$ and the results obtained here are consistent with that first report. The myod 1 early peak of expression agrees with the function of this transcription factor at the onset of the myogenesis in conjunction with the myod2, which classically appears more delayed than the myod $1^{29}$. The upregulation of myogenin at day 6 and the maintenance of high levels still at day 8 coincide with its role regulating the myoblast differentiation progress, as well as with the maximum levels of $m r f 4$ at day 8 , a factor more involved in the finalization and maturation of myotubes. Thus, the high parallelism between myogenin, myomaker and myomixer expression is quite clear and it is consequent with the role of these three factors on the later stages of myogenesis. In fact, the interaction of both fusogens with Myogenin could be explained by the presence of E-boxes in their promoters as pointed out in rainbow trout ${ }^{8}$.

Finally, the comparison of myomaker and myomixer transcript levels in fish at different ages suggests that both factors play a more active function at the stage of fingerlings. These results are in agreement with the findings described in rainbow trout 8,13 where the expression of myomixer and myomaker were maximum at the stage of embryo, decreasing progressively at 15, 150 and 1500 grams. All this information supports the role of both factors in somitogenesis or strong growing stages such us in fingerlings to decrease in juveniles or adults where the level of hyperplasia is less important. Thus, in mouse and zebrafish, the expression of myomixer declines soon after somitogenesis 9,15 , whereas in trout its expression is maintained throughout post-larval growth, i.e., in fry, juvenile and to a lesser extend in mature fish.

Overall, the present results support that myomaker and myomixer play in gilthead seabream an important role not only during developmental myogenesis, especially at the second part of the process, when the 
myocytes differentiation takes place, but also during regenerative myogenesis where their upregulation takes place only after 16 days of recovery, pointing out their role during the later differentiation stages. Therefore, our results contribute to understand the role of myomaker and myomixer in a fish species of undetermined growth, normally living at high temperature waters and with high interest for aquaculture.

\section{Declarations}

\section{Acknowledgements:}

This study was supported by the projects from the "Ministerio de Economía y Competi- tividad" (MINECO) AGL2015-70679-R and RTI2018-100757-B-I00 to J.G. and J.B., and the "Xarxa de Refèrencia d'R+D+I en Aqüicultura" and the 2017SGR1574 from the "Generalitat de Catalunya". M.P-A. and I.G-P., were supported by predoctoral fellowships (BES-2016-078697 and PRE2019-089578 respectively) from the "Ministerio de Economía y Competitividad" (MINECO) from the Spanish Government. F.M., was supported by a grant from the Department of Scholarship and Students' Affairs Abroad from the Ministry of Science, Research and Technology of I.R. Iran.

\section{Data availability}

All the data generated or analyzed during this study are included in this published article (and its Supplementary Material file).

Authors contributions: J.G. conceptualized the study; M.P-A.,V.J-P., I.G-P., A.S-M. and J.G. performed the sampling; M.P-A., V.J-P., A.O-T., I.G-P., and F.M., performed the laboratory analyses; M.P-A., V.J-P., A.O-T. and J.G. analyzed and interpreted the data; J.B., I.N., E.C. and J.G. acquired funding; M.P-A., V.J-P., A.O-T., J.C.G., I.N., E.C., J.F-B., C.J., G.D. and J.G. drafted and critically reviewed the manuscript. All authors read and approved the final paper. The authors have declared no conflict of interest.

\section{References}

1. Bentzinger, C. F., Wang, Y. X. \& Rudnicki, M. A. Building muscle: molecular regulation of myogenesis. Cold Spring Harb. Perspect. Biol. 4, a008342 (2012).

2. Ciciliot, S. \& Schiaffino, S. Regeneration of mammalian skeletal muscle: basic mechanisms and clinical implications. Curr. Pharm. Des. 16, 906-914 (2010).

3. Rossi, G. \& Messina, G. Comparative myogenesis in teleosts and mammals. Cell. Mol. life Sci. 71, 3081-3099 (2014).

4. Vélez, E. J. et al. Contribution of in vitro myocytes studies to understanding fish muscle physiology. Comp. Biochem. Physiol. Part - B Biochem. Mol. Biol. 199, 67-73 (2016).

5. Chen, B., You, W., Wang, Y. \& Shan, T. The regulatory role of Myomaker and Myomixer-MyomergerMinion in muscle development and regeneration. Cell. Mol. Life Sci. 77, 1551-1569 (2020).

6. Millay, D. P. et al. Structure-function analysis of myomaker domains required for myoblast fusion. Proc. Natl. Acad. Sci. 113, 2116-2121 (2016). 
7. Landemaine, A., Rescan, P. Y. \& Gabillard, J. C. Myomaker mediates fusion of fast myocytes in zebrafish embryos. Biochem. Biophys. Res. Commun. 451, 480-484 (2014).

8. Landemaine, A. et al. Trout myomaker contains 14 minisatellites and two sequence extensions but retains fusogenic function. J. Biol. Chem. 294, 6364-6374 (2019).

9. Bi, P. et al. Fusogenic micropeptide Myomixer is essential for satellite cell fusion and muscle regeneration. Proc. Natl. Acad. Sci. U. S. A. 115, 3864-3869 (2018).

10. Millay, D. P. et al. Myomaker is a membrane activator of myoblast fusion and muscle formation. Nature 499, 301-305 (2013).

11. Millay, D. P., Sutherland, L. B., Bassel-duby, R. \& Olson, E. N. Myomaker is essential for muscle regeneration. GENES Dev. 28, 1641-1646 (2014).

12. Quinn, M. E. et al. Myomerger induces fusion of non-fusogenic cells and is required for skeletal muscle development. Nat. Commun. 8, 1-9 (2017).

13. Perelló-Amorós, M., Rallière, C., Gutiérrez, J. \& Gabillard, J. C. Myomixer is expressed during embryonic and post-larval hyperplasia, muscle regeneration and fusion of myoblats in rainbow trout (Oncorhynchus mykiss). Gene 790, (2021).

14. Zhang, H. et al. Human myotube formation is determined by MyoD-Myomixer/Myomaker axis. Sci. Adv. 6, 1-14 (2020).

15. Shi, J. et al. Requirement of the fusogenic micropeptide myomixer for muscle formation in zebrafish. Proc. Natl. Acad. Sci. 114, 11950-11955 (2017).

16. Louro, B., Marques, J. P., Power, D. M. \& Canário, A. V. M. Having a BLAST: Searchable transcriptome resources for the gilthead sea bream and the European sea bass. Mar. Genomics 30, 67-71 (2016).

17. García de la serrana, D. et al. Characterisation and expression of myogenesis regulatory factors during in vitro myoblast development and in vivo fasting in the gilthead sea bream (Sparus aurata). Comp. Biochem. Physiol. - A Mol. Integr. Physiol. 167, 90-99 (2014).

18. Vélez, E. J. et al. IGF-I and amino acids effects through TOR signaling on proliferation and differentiation of gilthead sea bream cultured myocytes. Gen. Comp. Endocrinol. 205, 296-304 (2014).

19. Montserrat, N., Gabillard, J. C., Capilla, E., Navarro, M. I. \& Gutiérrez, J. Role of insulin, insulin-like growth factors, and muscle regulatory factors in the compensatory growth of the trout (Oncorhynchus mykiss). Gen. Comp. Endocrinol. 150, 462-472 (2007).

20. Bustin, S. A. et al. The MIQE Guidelines: Minimum Information for Publication of Quantitative RealTime PCR Experiments. Clin. Chem. 55, 611-622 (2009).

21. Salmerón, C., Navarro, I., Johnston, I. A., Gutiérrez, J. \& Capilla, E. Characterisation and expression analysis of cathepsins and ubiquitin-proteasome genes in gilthead sea bream (Sparus aurata) skeletal muscle. BMC Res. Notes 8, 149 (2015).

22. Pfaffl, M. W. A new mathematical model for relative quantification in real-time RT-PCR. Nucleic Acids Res. 29, 45e - 45 (2001).

23. Zhang, B., Su, Y., Zhou, J., Zheng, Y. \& Zhu, D. Toward a Better Regeneration through Implant-Mediated Immunomodulation: Harnessing the Immune Responses. 2100446, 1-19 (2021). 
24. Perelló-Amorós, M. et al. Ghrelin and Its Receptors in Gilthead Sea Bream: Nutritional Regulation. Front. Endocrinol. (Lausanne). 9, 1-14 (2018).

25. Rowlerson, A., Radaelli, G., Mascarello, F. \& Veggetti, A. Regeneration of skeletal muscle in two teleost fish: Sparus aurata and Brachydanio rerio. Cell Tissue Res. 289, 311-322 (1997).

26. Rescan, P. Y., Ralliere, C., Lebret, V. \& Fretaud, M. Analysis of muscle fibre input dynamics using a myog:GFP transgenic trout model. J. Exp. Biol. 218, 1137-1142 (2015).

27. Perelló-Amorós, M. et al. Diet and Exercise Modulate GH-IGFs Axis, Proteolytic Markers and Myogenic Regulatory Factors in Juveniles of Gilthead Sea Bream (Sparus aurata). Animals 11, (2021).

28. Schmidt, J. G., Andersen, E. W., Ersbøll, B. K. \& Nielsen, M. E. Muscle wound healing in rainbow trout (Oncorhynchus mykiss). Fish Shellfish Immunol. 48, 273-284 (2016).

29. Tan, X. \& Jun Du, S. Differential expression of two MyoD genes in fast and slow muscles of gilthead seabream (Sparus aurata). Dev. Genes Evol. 212, 207-217 (2002).

\section{Figures}

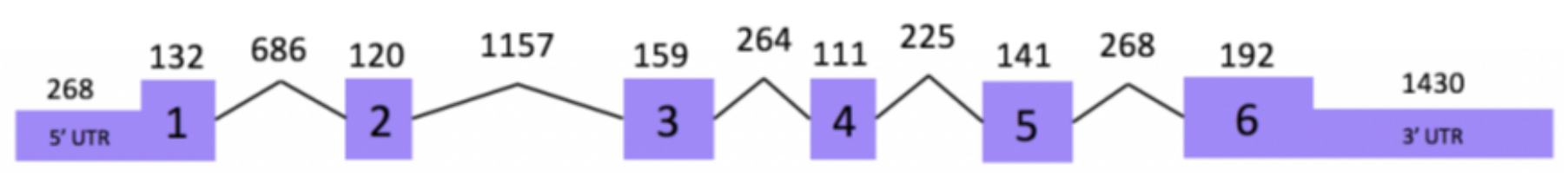

\section{Figure 1}

Structure of the $S$. aurata myomaker gene. The size of exons including the UTRs (purple boxes) and introns (lines) are indicated in number of nucleotides. 


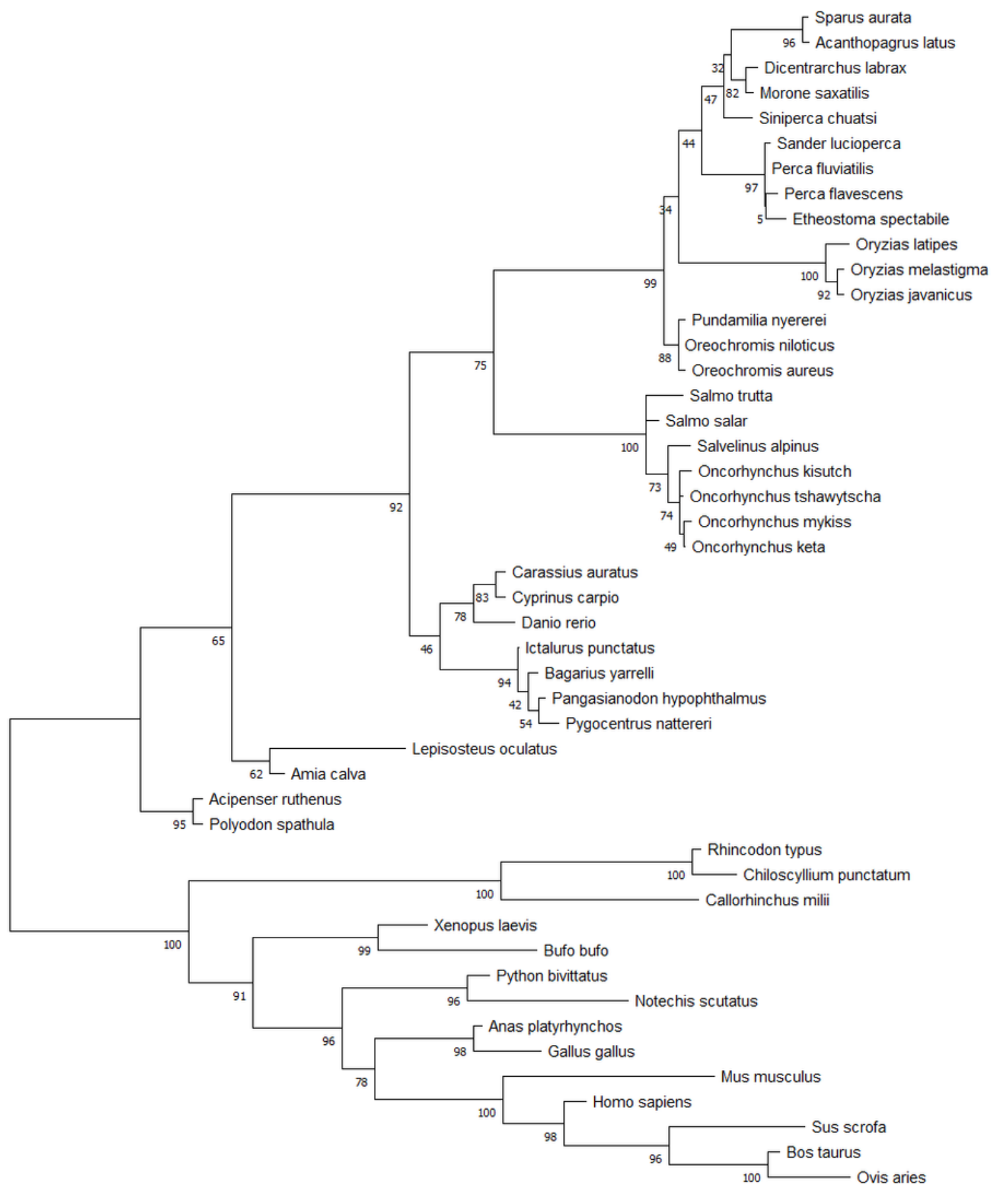

0.10

Figure 2

Phylogenetic analysis of Myomaker protein sequences among mammals, birds, reptiles, amphibians, and fish. Multiple alignment of whole protein sequences was done through the MAFFT tool (https://mafft.cbrc.jp/alignment/server/) with the iterative refinement L-INS-i method. The phylogenetic tree was developed with the Maximum Likelihood phylogeny and the JTT+G substitution model using the 
MEGA $11 \mathrm{v} 11.0 .1 .10$ program. The numbers in the tree nodes represent the percentage of the bootstrap values after 500 replicates.

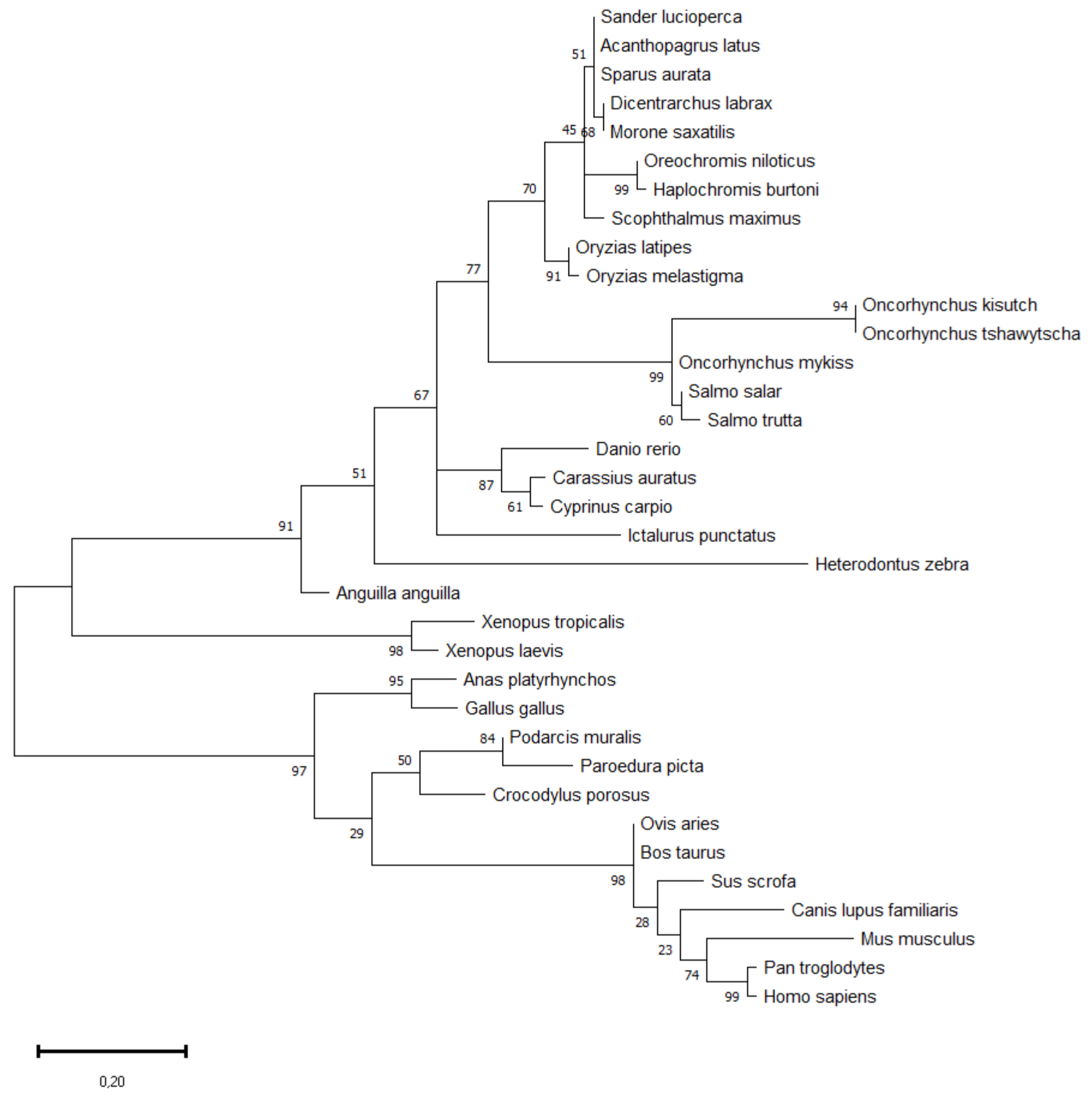

Figure 3

Phylogenetic analysis of Myomixer protein in mammals, birds, reptiles, amphibians, and fish. Multiple alignment of whole protein sequences was done through the MAFFT tool 
(https://mafft.cbrc.jp/alignment/server/) with the progressive G-INS-1 method. The phylogenetic tree was developed with the Maximum Likelihood phylogeny and the JTT+G substitution model using the MEGA X 11 v11.0.1.10 program. The numbers in the tree nodes represent the percentage of the bootstrap values after 500 replications.

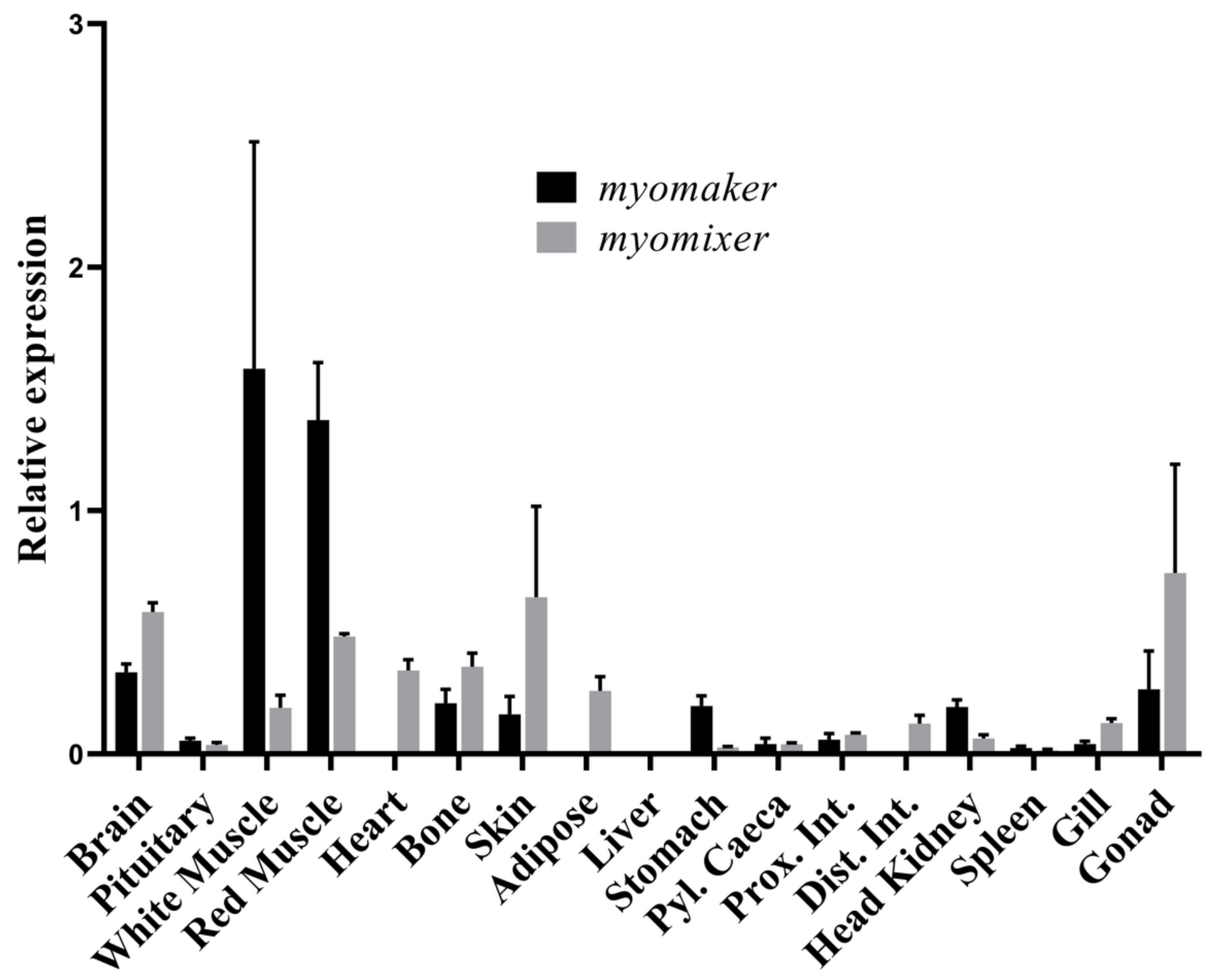

Figure 4

myomaker and myomixergene expression in several tissues of $200 \mathrm{~g}$ gilthead sea bream. Data are represented as means \pm SEM $(n=4)$. 

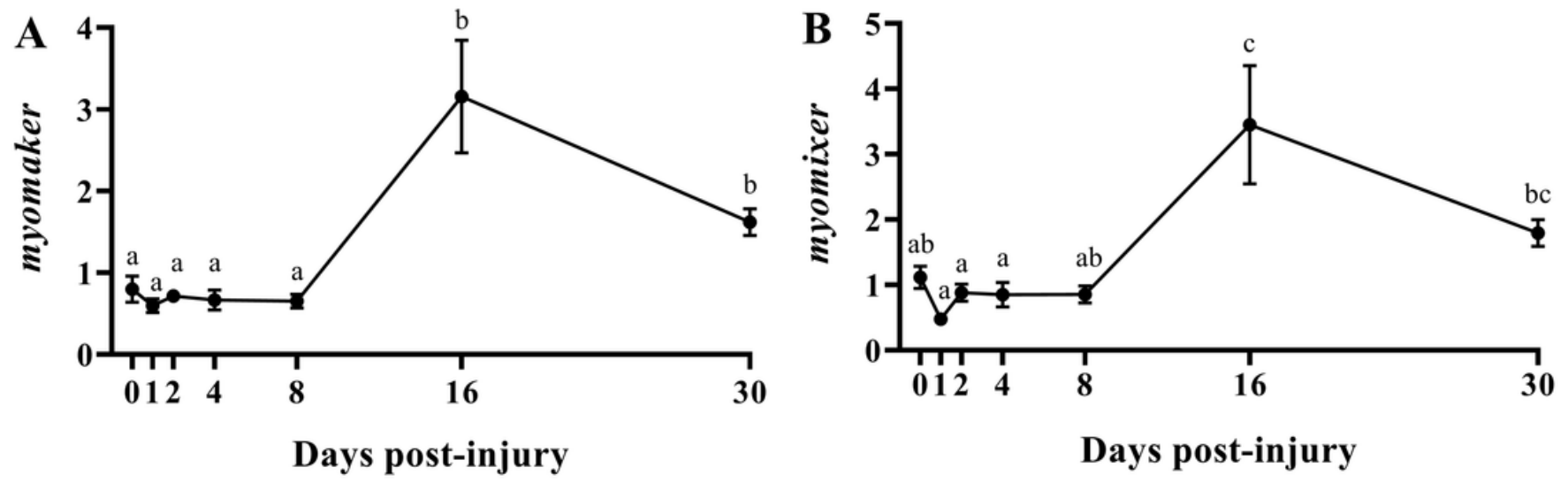

Figure 5

Relative gene expression of myomaker (A) and myomixer (B) along the regeneration experiment in white skeletal muscle from day 0 (time of injury) to day 30 . Data are presented as means \pm SEM $(n=10)$. Different letters indicate significant differences $(p<0.05)$. 

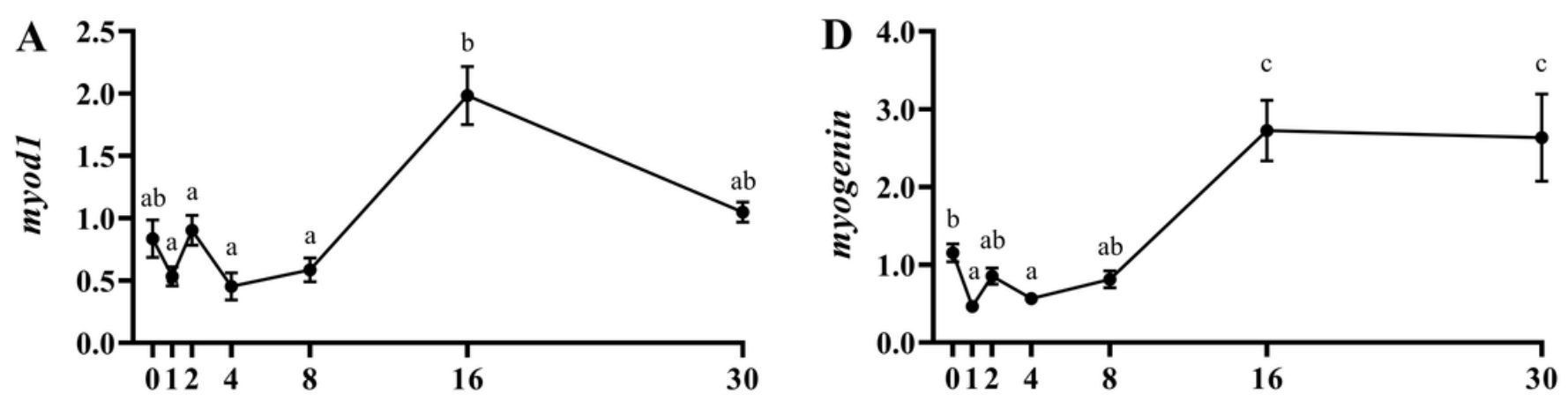

Days post-injury

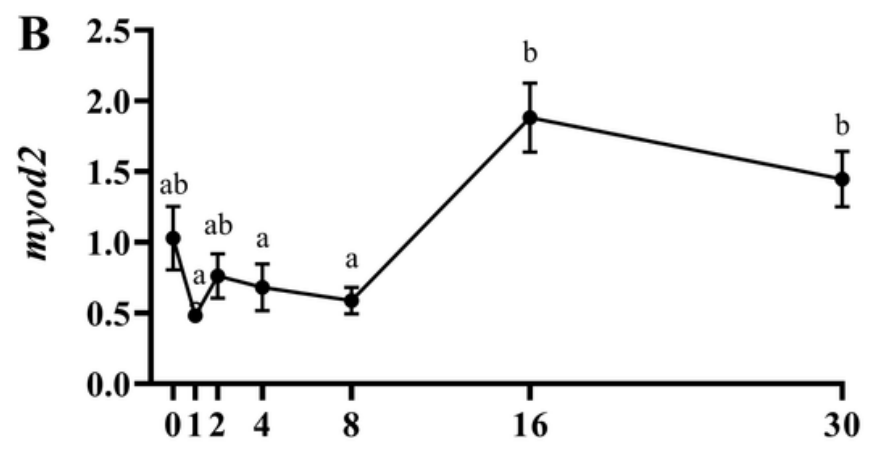

Days post-injury
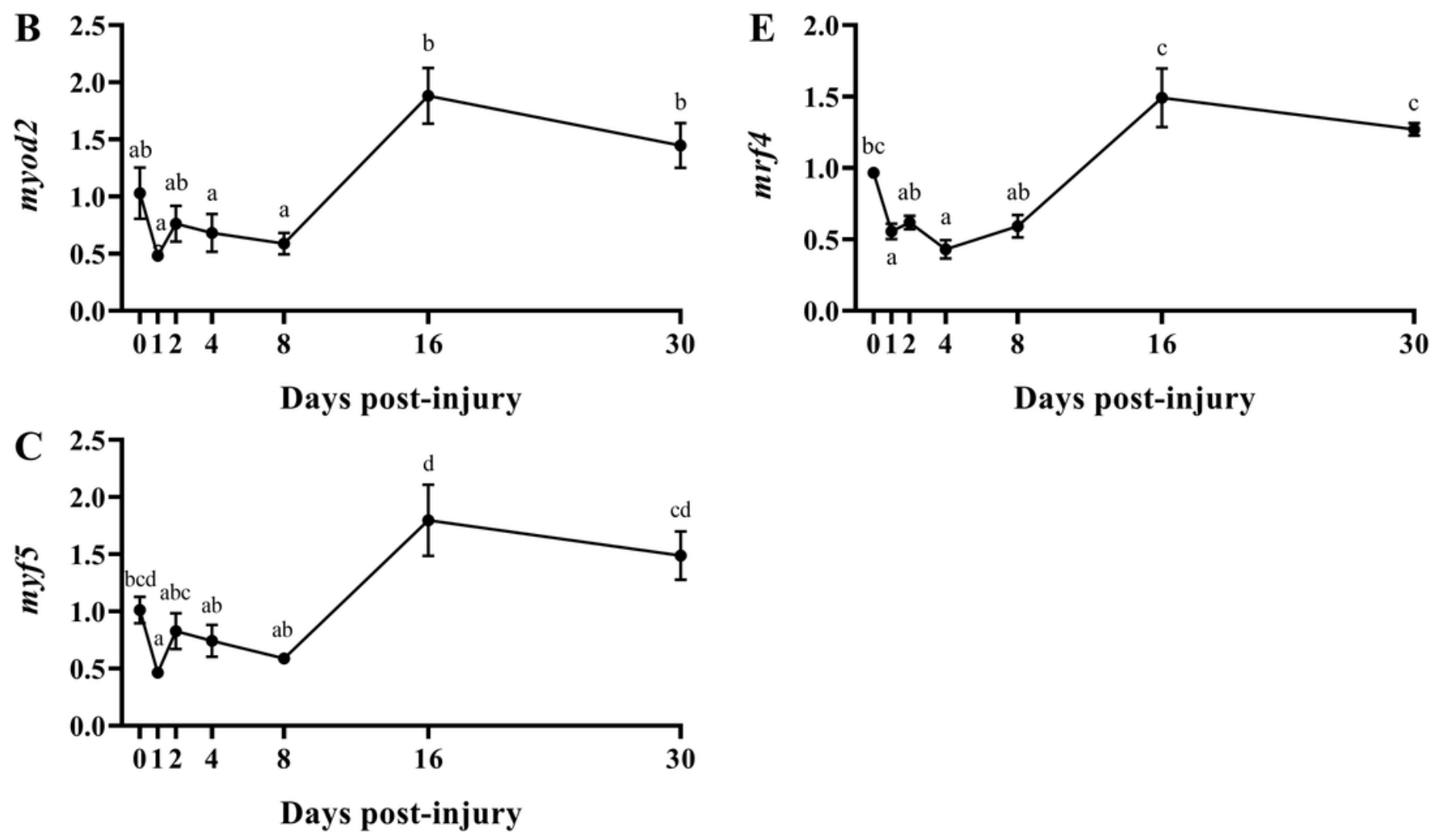

Days post-injury

\section{Figure 6}

Relative gene expression of the myogenic regulatory factors (MRFs) along the regeneration experiment in white skeletal muscle from day 0 (time of injury) to day 30 . Data are presented as means $\pm \operatorname{SEM}(n=10)$. Different letters indicate significant differences $(p<0.05)$. 


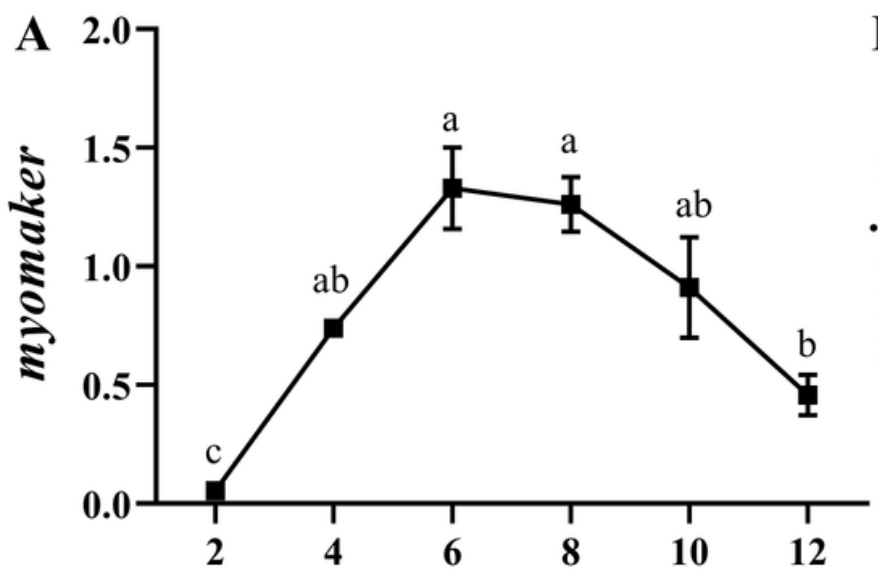

Days in culture

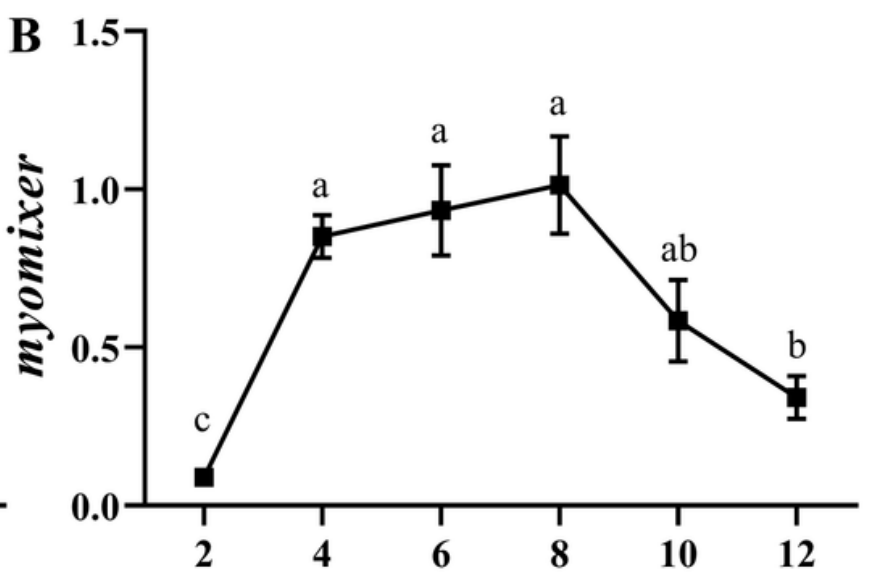

Days in culture

\section{Figure 7}

Relative gene expression of myomaker (A) and myomixer (B) along the primary culture of myoblasts. Data are presented as means \pm SEM $(n=6)$. Different letters indicate significant differences $(p<0.05)$. 

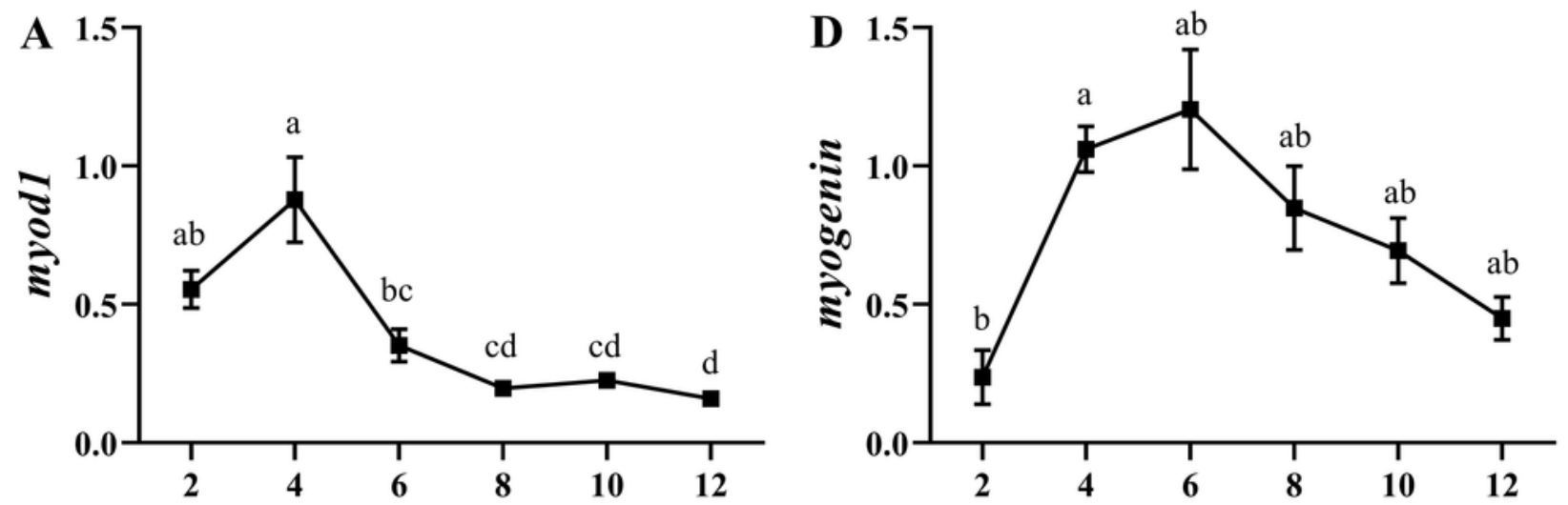

Days in culture
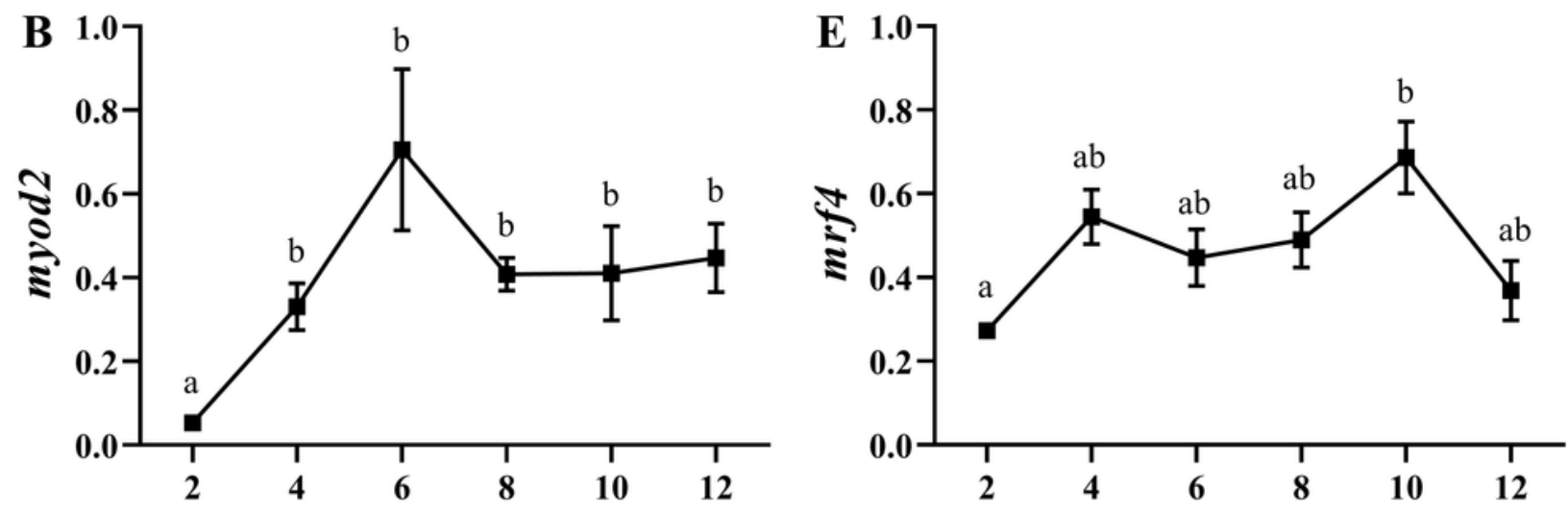

Days in culture

\section{Days in culture}

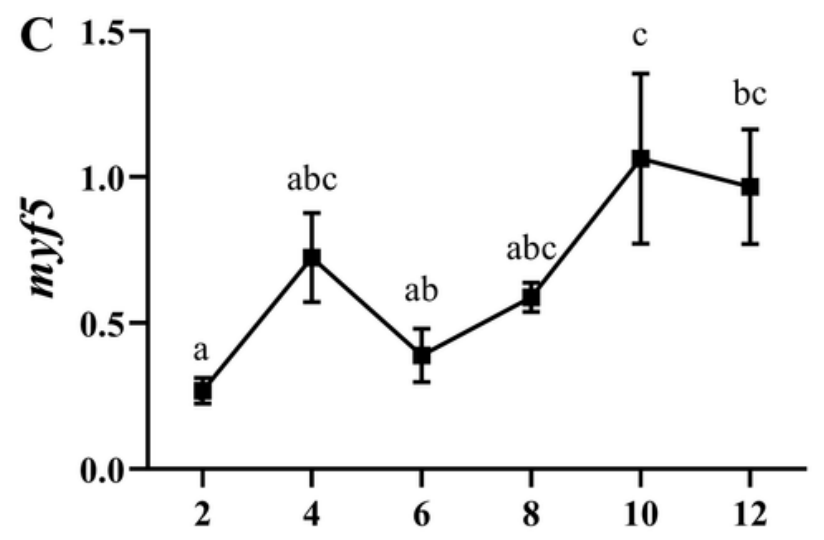

\section{Days in culture}

Figure 8

Relative gene expression of the myogenic regulatory factors (MRFs) along the primary culture of myoblasts. Data are presented as means \pm SEM $(n=6)$. Different letters indicate significant differences $(p<0.05)$. 



Figure 9

Relative gene expression of myomaker and myomixer in white skeletal muscle of three different sizes of gilthead sea bream. Data are presented as means \pm SEM $(n=6)$. Different letters indicate significant differences $(p<0.05)$.

\section{Supplementary Files}

This is a list of supplementary files associated with this preprint. Click to download.

- SUPPLEMENTARYMATERIALPAPERMYOMAKERANDMYOMIXERSEABREAMresubmitted14.12.21.docx 